\title{
THE IMPORTANCE OF SLEEPING POSTURE IN THE SPREAD OF PULMONARY TUBERCULOSIS
}

\author{
BY \\ W. H. HELM \\ From the London Chest Hospital
}

(RECEIVED FOR PUBLICATION AUGUST 22, 1951)

When an infective pulmonary condition spreads from one lung to the other the process is frequently spoken of as a "spill-over." The mechanism implied is that of bronchial aspiration of infected material from the originally affected lung to the other. This may occur in a number of diseases, including pulmonary tuberculosis, empyema with bronchopleural fistula, lung abscess, and bronchieciasis. The present investigation is confined to its occurrence in pulmonary tuberculosis.

It has long been recognized that there are definite areas in which tuberculous contralateral spreads are most likely to occur and that the lesions produced have a characteristic radiological appearance. Landreth and Morlock (1928) were impressed by the frequency with which these lesions occurred in the middle zones, and with the constancy of the radiological picture, which they described as patches of bronchopneumonia occupying a wedge-shaped area, the base of the wedge being at the periphery and the apex towards the hilum. The middle zones have also been named by Wu and T'Ang (1937), Fraenkel (1942), Rubin (1947), and Kayne, Pagel, and O'Shaughnessy (1948) as areas frequently affected. More detailed localization has been undertaken by Cardis and Joannette (1930), who identified the areas as the inferior and external margins of the upper lobes, and by Dufourt and Brette (1947) as, on the left, the lingula, and, on the right, the pectoral segment and axillary area.

Bronchial aspiration of infected material has been broadly accepted by these authors as the mechanism of contralateral spread, but few explanations have been given for the remarkable constancy of the areas affected. Rubin suggests that the frequent occurrence of spread of the disease from the right upper lobe to the left middle zone is due to the slope of the bronchi favouring the contralateral aspiration of "caseous liquid" sputum. Cardis and Joannette believe that the localization of the lesions is due to the greater pulmonary elasticity in these areas facilitating bronchial aspiration. Wu and T'Ang, in a study of 242 cases of pulmonary tuberculosis with contralateral spread, conclude that gravity in the presence of a cough reflex depressed by sedatives or sleep is the cause of the spread and the explanation of its localization.

\section{Present Investigation}

The cases in the present series are based on a review of the 1,200 in-patients at four sanatoria and a chest hospital. Two groups were considered to be possible 
sources of information regarding the mechanism of spread. They were cases with unilateral cavernous disease and a bronchogenic contralateral spread (157), and contrasting with these cases with unilateral cavernous disease and a radiologically clear contralateral lung (93). The distribution of the spread lesions in 157 cases is shown below.

For convenience the term "axillary sub-segments" is used to refer to the areas supplied by the lateral branches of the anterior and posterior divisions of the upper lobe bronchi.

TABLE I

Distribution* of Bronchogenic Contralateral Spreads

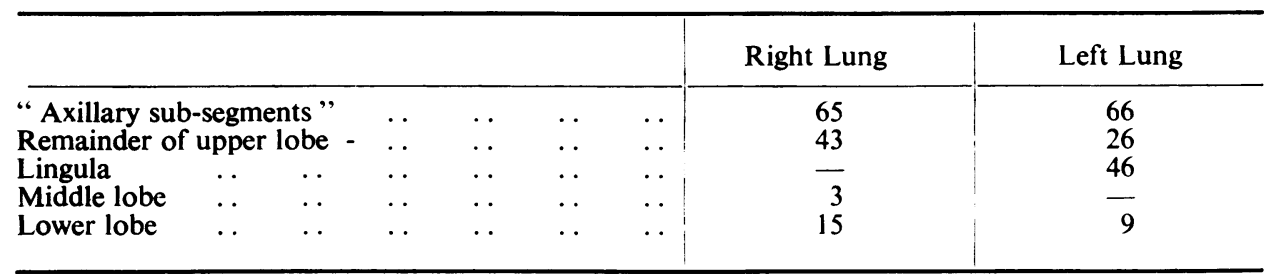

* Where more than one area is significantly affecied in any one case each is included.

It may be argued that the lesions recorded here as contralateral spreads are not in fact bronchogenic infections due to aspiration, but are merely reactivations of haematogenous foci previously invisible radiologically. In any one particular instance it would be difficult to deny that such might be the case. Many instances were encountered where this was the probable aetiology of a lesion appearing in the previously clear lung. These cases were excluded from the series, as also were all those with radiological evidence of this type of lesion in addition to a spread. The evidence against a large proportion of the lesions in the present series being reactivations is strong ; the distribution is not that found in such foci and the radiological appearance is typical of a bronchogenic infection, the lesions being segmental and frequently accompanied by areas of atelectasis or segmental inflammatory changes. The very frequent association of spread in the lingula and left upper lobe is also in favour of a bronchogenic infection, the lingular bronchus being the first branch of the left upper lobe bronchus whereas the middle lobe is very rarely affected, the bronchus being entirely separate from that of the right upper lobe. The evolution of the lesions is different from that of haematogenous foci. They are comparatively benign, and frequently clear quickly without treatment and dramatically with chemotherapy. They cavitate infrequently and their fate appears to be mainly dependent on that of the major lesion in the other lung (Fraenkel, 1942 ; Dufourt and Brette, 1947).

It may also be argued that these lesions are non-tuberculous and due to aspiration of secretions containing other organisms, or simply to the mechanical blockage of bronchi. Both these processes undoubtedly occur and may account for the very rapid clearing seen in some cases. The sputum was, however, positive in every case in this series and healing by calcification was frequently seen.

The "axillary sub-segments" and lingula are the areas principally affected. When the whole upper lobe is involved the "axillary sub-segments" are usually the first and the most severely affected. These are the areas to which aspirated 
material is most likely to gravitate when the patient lies on his side (Brock, 1946). In view of this and the fact that lateral posture is adopted principally during sleep when other conditions are also most favourable for bronchial aspiration, the relation between sleeping posture and contralateral spreads was investigated. Both groups of patients were questioned in the following manner.

They were first asked, “In what position do you sleep at night ?" If the reply was, "I don't know," or "Any way," or "I turn about," then no further question was asked, and their posture was classified as " any way." If the answer was, "Left side," or "Right side," or "Back," they were further questioned as to whether this had always been so and as to whether or not the position was always maintained. Those who started on one side and then turned were classified as " any way." Those stating a definite side were questioned as to whether they knew why they slept in that particular position. Many said that they were simply more comfortable that way, but others gave such answers as, "I used to face the wall at home," or "Because I am deaf in the other ear." In this series surprisingly few patients said that they slept on their backs, and none considered that they constantly maintained this position.

The answers given by both groups of patients are shown in Table II.

TABLE II

Sleeping Posture in Patients with and without Spread

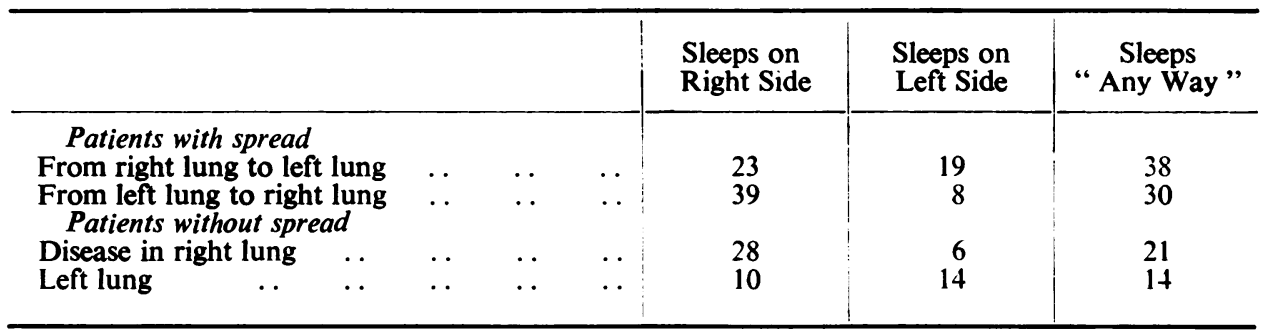

These results can be summarized thus (Table III).

TABLE III

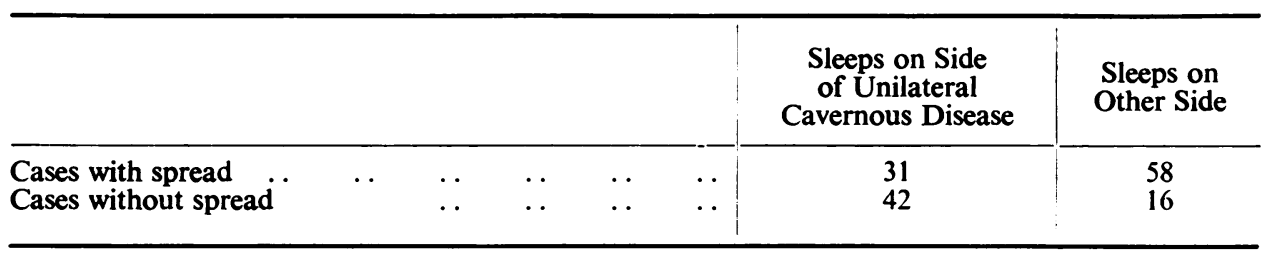

Applying Fisher's test of this table, with Yates's correction, the value of $\chi^{2}$ is found to be 18.4, which is highly significant. The chances of exceeding such a deviation in the right direction are less than 1 in 10,000 . This means that it is highly probable if not certain that the sleeping side is a factor in the incidence of spread. 


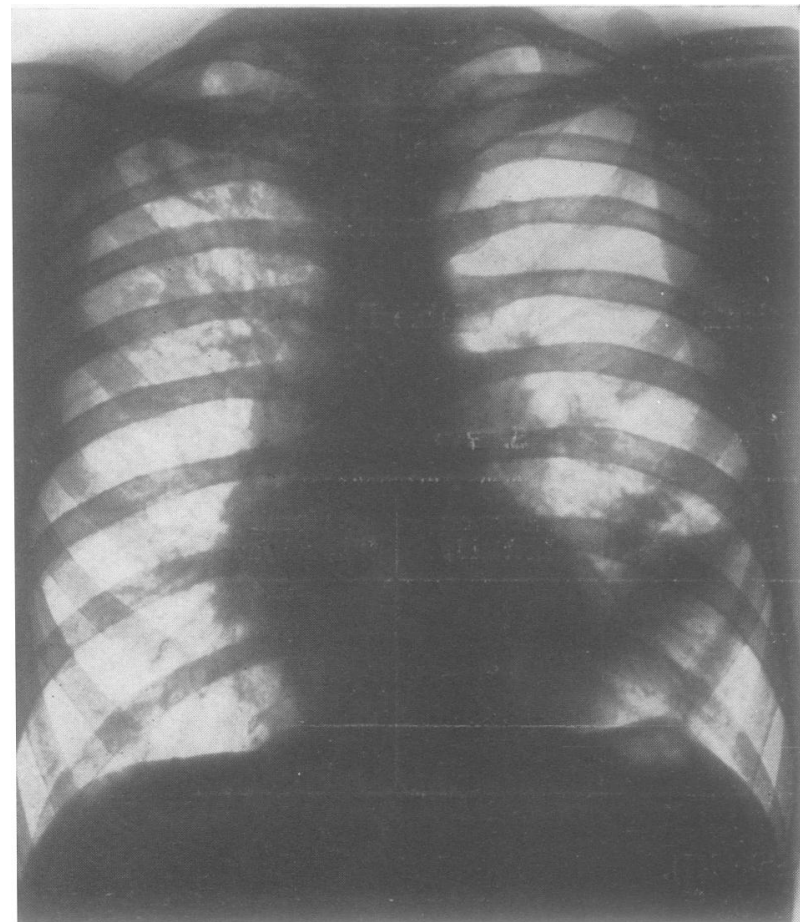

FIG. 1.-Right upper lobe of Case 1 showing cavernous tuberculosis with a lingular spread.

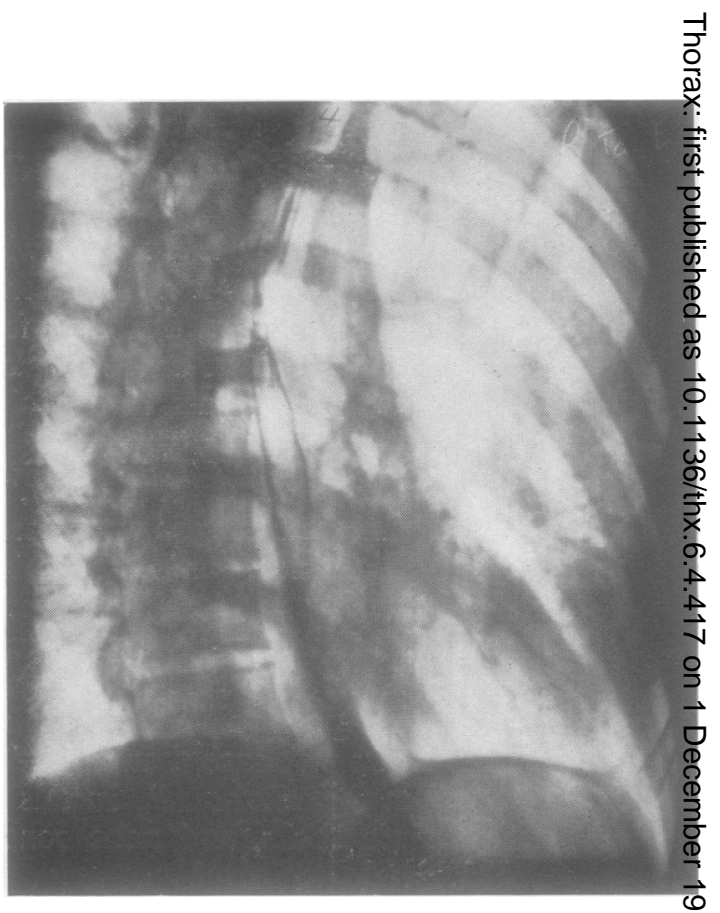

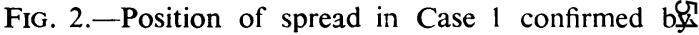
oblique view.

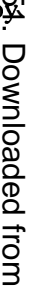

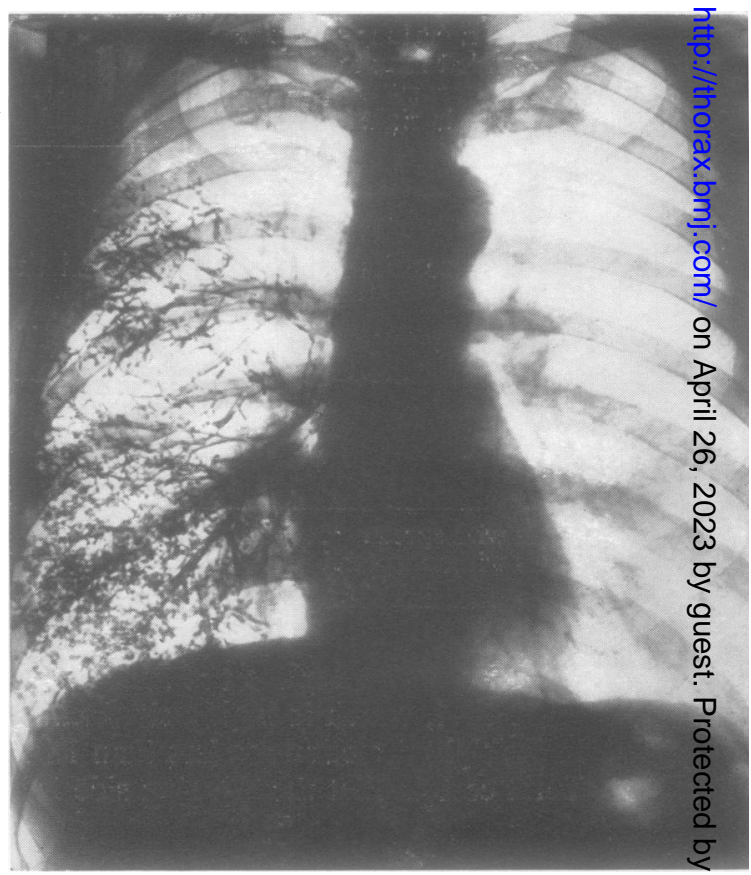

Fig. 3.-Right unilateral bronchogram of Case 


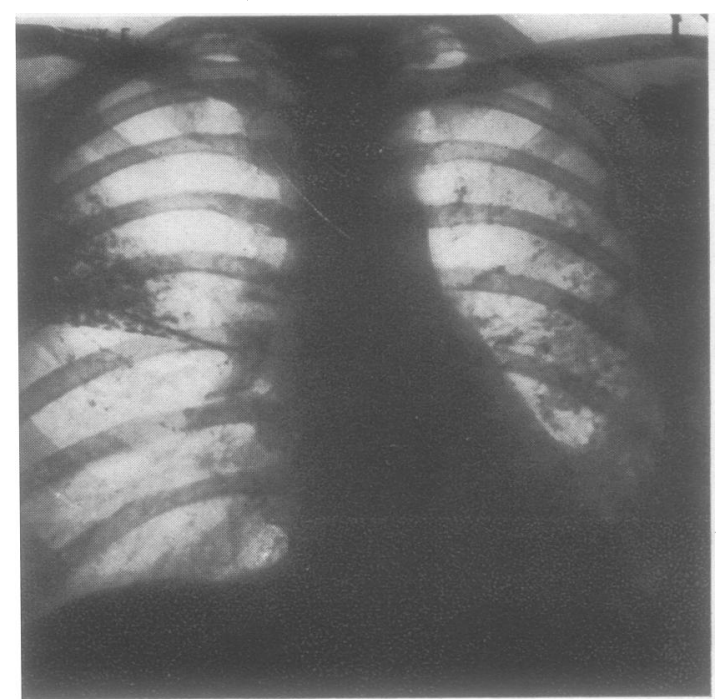

7.-Radiograph of Case 3 to show spill-over to the right "axillary" sub-segment after a night's sleep on the right side.

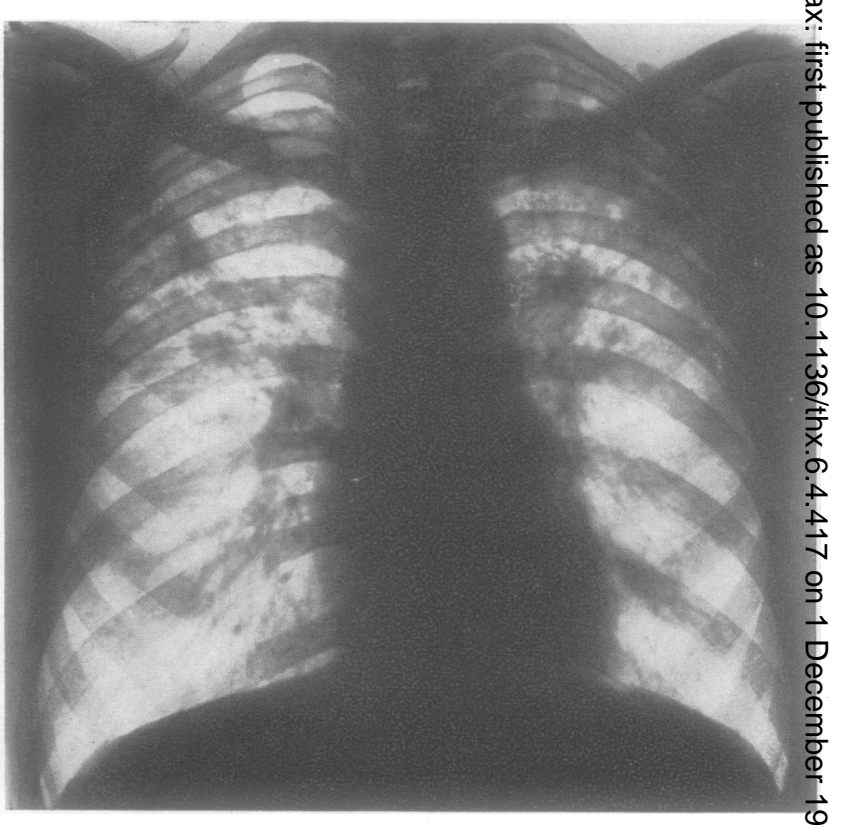

FIG. 8.-Radiograph of Case 4 showing left upper lober cavernous tuberculosis and a right "axillary sub-segment spread.

It was felt that possibly too much reliance was being placed on the patients' own ideas regarding their sleeping posture. Twelve patients who stated a definite side were therefore observed at hourly intervals between the hours of 11 p.m. and 5 a.m. for one week. In 500 out of 588 observations the posture was as stated. In 69 instances patients were on their backs, and in 19 on the opposite side to that stated.

\section{EXPERIMENTAL EVIDENCE}

Some experimental support for the lateral posture-gravitation-aspirationtheory of the spread mechanism was obtained by Wu and T'Ang (1937), who carried out bronchograms and found that radio-opaque oil spread easily from one upper lobe to the other in this posture and that the oil entered the "spread" areas.

Although iodized oil and tuberculous sputum are not entirely comparable substances they are both capable of gravitation and aspiration without complete expulsion by cough. A series of simple experiments has, therefore, been carried out to study the effect of posture on the spread of oil from one lung to the other. In 11 cases unilateral bronchograms were obtained, and the patients then lay on their oil-free sides for one hour or longer and further radiographs were taken. In 10 cases a spill-over of oil had occurred to the "spread" areas. In no case was there more than minimal alveolar filling in any other area, and in only three was there a trace of oil in the lower lobe bronchus. The maximum spill-over occurred during sleep in the lateral posture. These results were obtained both with and without coughing, and were apparently similar whether or not the bronchial mucosa was anaesthetized.

In two cases similar contralateral spills were obtained after injection of iodized oil into empyema cavities with bronchopleural fistulae, and in one instance oil was 
injected into a non-tuberculous upper lobe cavity with the same result. In five cases an attempt was made by encouraging energetic coughing to obtain a contralateral spread of oil when the patient was lying on the oil-containing side. In no case was any spread obtained. With the patient in the erect posture, sitting or standing, no spill-over occurred without coughing (five cases), but with coughing a spill to the other lobe occurred in three out of five cases. No oil entered the "spread" areas in this posture.

It may, therefore, be said that iodized oil can, and usually does, spread from one lung to the "axillary sub-segments" (and lingula) of the other with or without coughing when the patient lies with the oil-containing side uppermost. It seldom spreads in significant quantity to any other part of the lung in this posture, and it is probable that a similar spread is most unlikely to occur with the patient in any other position.

\section{Discussion}

It seems likely that tuberculous sputum in the bronchial tree initiates a cough reflex more readily than oil and is, therefore, more likely to be completely expelled except when the reflex is depressed during sleep or anaesthesia. In unusual circumstances, however, contralateral aspiration of tuberculous material undoubtedly occurs in the absence of a depressed cough reflex, and the area affected is again related to the patient's posture. This is seen in bronchopleural fistulae and haemoptysis where spread to the opposite side is frequent. In both these conditions the circumstances are not entirely comparable to those present during the period of development of the usual contralateral spread because the inflow to the bronchial tree overwhelms the cough mechanism and some degree of aspiration is almost certain to occur. The rarity of lower lobe spread in the present series supports the contention that in normal waking circumstances the cough mechanism is able to prevent contralateral aspiration of sputum since a considerable period of the day is spent in a posture favouring lower lobe aspiration.

If lateral posture and a depressed cough reflex are accepted as essentials for contralateral spread, it may still seem surprising that the lower and middle lobes are so rarely involved. Once the infected material had gravitated or been coughed across from one side and into the main bronchus of the other, it might be expected that aspiration into the lower lobes would occur fairly frequently. It is possible that the reason this is seldom found is that with the patient in the lateral posture the sputum descends along the lateral wall of the main bronchus as oil has frequently been shown to do ; the first bronchial orifice it then encounters is that of the upper lobe, where gravity and aspiration allow descent into this opening and on into the most dependent parts, the "axillary sub-segments" and lingula. If the material passes the bronchial opening and continues down the lateral wall of the main lower lobe bronchus, any expulsive force, cough, or ciliary action will impel it back past the upper lobe orifice once more to run the double hazard of gravity and aspiration. It is probable that in the lateral posture the cough meshanism is considerably more effective for the lower than the upper lobe in the dependent lung. Owing to the anatomical arrangement of the bronchial tree thene is in the lower lobe minimal gravity to overcome compared with the maximal force in the upper lobe. The 
action, in coughing, of the abdominal muscles and intra-abdominal pressure on the diaphragm may have a more direct expulsive force for the lower than the upper lobe. Sleep may well accentuate these disparities.

The infrequency of middle lobe involvement is readily understood if the essential part played by lateral posture in the mechanism is kept in mind.

\section{Conclusion and ReCOMmendation}

The importance of sleeping posture in pulmonary inhalation infections is well recognized. Brock (1946) stresses its significance in the aetiology of lung abscess, and suggests that it may be a factor in pulmonary tuberculosis. In previous investigations concerning contralateral spread Dufourt and Brette (1947) and $\mathrm{Wu}$ and T'Ang (1937) have drawn attention to the importance of sleeping posture, and $\mathrm{Wu}$ and T'Ang strongly recommend that patients with unilateral disease should sleep on the affected side. Dillwyn Thomas (1951) believes in the use of posture both for cavity closure and also for preventing bronchogenic spread. In spite of the scarcity of medical literature on the subject it is not uncommon to find that patients have received such instructions regarding sleeping posture from their chest physicians or at their sanatoria. Similar recommendations are made in some of the lay literature for tuberculous subjects. The idea appeals to most patients as a simple and commonsense means of protecting the good lung. The majority of patients interviewed in this investigation had received no such instructions.

The evidence is strong that tuberculous contralateral bronchogenic spread is a definite and usually easily recognizable entity. It is clear that sleeping posture is of aetiological significance, and it seems likely that in normal circumstances contralateral spread occurs almost entirely during sleep in the lateral posture. Conversely in normal circumstances contralateral spread is most unlikely to occur if the patient sleeps on the side of the unilateral disease.

It is therefore recommended that all patients with unilateral pulmonary tuberculosis should be made to sleep on the affected side. This measure alone would protect only the opposite lung, and bronchogenic spread to other parts of the same side could still occur. The safest procedure would therefore be the adoption during sleep of the position in which the major lesion would be most dependent, the sputum thereby being retained in the already infected area until a fully sensitive and unimpeded cough mechanism could expel it completely. In selected cases this posture could be maintained with advantage throughout the 24 hours for a period of weeks or more, thereby both preventing bronchogenic spread and treating the cavity by the method of postural dependency described by Dillwyn Thomas (1951).

\section{SUMMARY}

One thousand two hundred in-patients with pulmonary tuberculosis were reviewed.

Those with typical contralateral bronchogenic spreads and those with unilateral cavernous disease and a radiologically clear contralateral lung were further investigated. The areas predominantly affected by the spreads suggest that lateral posture is a factor in the aetiology. This position is adopted principally during sleep when 
conditions are most favourable for bronchial aspiration. The relation of sleeping posture to spread was therefore investigated and found to be significant.

Recommendations regarding sleeping posture are made.

I wish to thank the consultant staff of the London Chest Hospital and the medical superintendents of Kettlewell, Lenham, and Preston Hall Sanatoria and the National Sanatorium, Benenden, for their permission to investigate their cases and for their assistance; Dr. H. V. Morlock for his advice and encouragement throughout, and Dr. E. W. Street for much assistance in the early work; Drs. K. W. Hinson, N. Lloyd Rusby, and J. G. Scadding for criticism and suggestions; the members of the nursing profession who have played a considerable part in the investigation; and finally Miss Craddock, of the secretarial staff of the London Chest Hospital, who has worked with me throughout.

\section{REFERENCES}

Brock, R. C. (1946). Anatomy of the Bronchial Tree. London.

Cardis, F., and Joannette, A. (1930). Rev. Tuberc., Paris, 3 ser, 11, 83.

Dufourt, A., and Brette, R. (1947). J. Med. Lyon, 28, 899.

Fraenkel, E. (1942). Tubercle, Lond., 23, 227.

Kayne, G. G., Pagel, W., and O'Shaughnessy, L. (1948). Pulmonary Tuberculosis, 2nd ed. London. Landreth, J. F., and Morlock, H. V. (1928). Tubercle, Lond., 10, 101.

Rubin, E. H. (1947). Diseases of the Chest. Philadelphia.

Thomas, D. M. E. (1951). Personal communication.

Wu, C., and T'Ang, B. H. Y. (1937). Amer. J. Roentgenol., 37, 180. 